\title{
Design and Development of Route-selection and Decision-making System
}

\author{
Junfeng Zhang \\ School of Resource and Environment \\ North China University of Water Resources and Electric \\ Power \\ Zhengzhou 450011, People R China \\ E-mail: zh-junfeng@163.com
}

\author{
Yan Shen \\ School of Resource and Environment \\ North China University of Water Resources and Electric \\ Power \\ Zhengzhou 450011, People R China \\ E-mail: yunwuigirl@163.com
}

\begin{abstract}
In the study of route selection, due to the diversity, fuzziness, no-determinacy and randomness of the evaluating index, it is very complex for the information processing and decision making, and the CAD system cannot solve these questions at present. This paper aims at the facts of Tianshan-Road, utilizes ArcEngine components of ESRI Company and imports correlative theories, methods and technique of GIS to design the preliminary project based on several mathematical model, such as fuzzy comprehensive evaluation, multi-regress, and information model. This system can offer powerful basis of route-selection and decision-making in Tianshan-Road.
\end{abstract}

Keywords- tianshan road; vector overlap; region stability; route-selection

\section{INTRODUCTION}

The social from all walks of life pay more and more attention to highway traffic as the artery of the national economy ${ }^{[1]}$. At present, the important factors that affect the Chinese highway designing level and efficiency are that there is no breakthrough in the traditional designing pattern and method, especially lack of high-tech content. Although we have developed a number of highway route CAD system, their functions mainly place emphasis on calculation and draw, and are short of interactions with designers effectively in decision-making, such as scientific route-selection and spatial analysis ${ }^{[2-4]}$. Based on these, this paper introduces the related theories, methods and models of GIS, especially the spatial data analysis ability, through theoretical analysis, model-building, selecting impact factor and calculating region stability, to build a route-selection system and provide powerful support for the initial determination of scheme.

\section{TECHNOLOGY PRINCIPLE OF ROUTE SELECTION}

Region stability along the road is a very important factor which decides the route selection of highway. Routing should fully consider the geological complexity and diversity for bypassing the bad geological section and reducing the mileage through them ${ }^{[5]}$. Based on this, the first step in the routing is to analyze and calculate the region stability along the road. The theory basis of region stability is the engineering geological analogy method, which is according to the existing topographic variables related to disaster spatial distribution, to predict the spatial distribution of future disasters. Using GIS analytical method for stability assessment is that consider each factor which affects the region on the stability as a vector layer, through the overlay and relevant mathematical model, to speculate other unknown stability from the known ones. The results will be an important evaluation index of route selection.

The paper's major strategy is weighted cost method: firstly uniform and rasterize effect factors, secondly overlay the factors to get weighted cost, and then input the route beginning to obtain the least distance cost and direct cost toward the beginning, finally input the route end to obtain optimal path through the cost raster-data. In order to take the truth into account, the user can also input feature points in the design, such as city and town, which the route will pass, the system will automatically generate an optimal path passing the certain points by loop computing.

\section{Design OF OVERALl ARCHITECTURE}

A. System Workflow

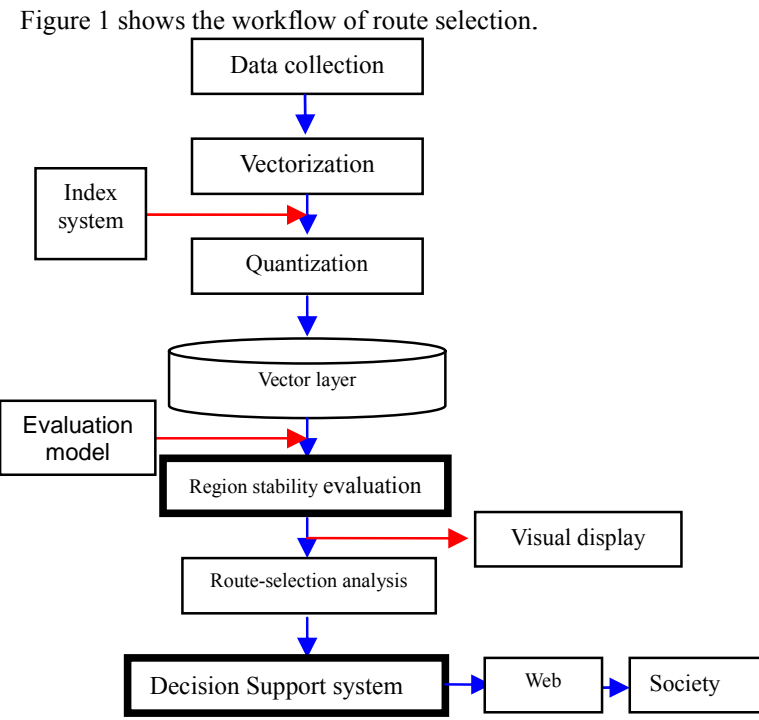

Figure 1. The workflow of Route-selection of Tianshan-Road

\section{B. The characteristics of system development}

(1) By using seamless developing way, the system combines function of ArcGIS through secondary development component "ArcEngine", to make it as application-oriented, flexible and professional highway construction software. ArcEngine based on ArcObjects, but has stronger development capabilities and independence; 
meanwhile, it breaks away from the ArcGIS completely. Taking advantage of the ArcEngine, we can embed the secondary development product into our customized project easily.

(2) This system combines the strong spatial analysis functions of GIS with a variety of mathematical models, for example fuzzy comprehensive evaluation, multi-regress, and information model, to evaluate the GIS region stability. This combination extends the spatial analysis ability of GIS in the field of mathematical model further, and achieves the purpose of multidisciplinary cross-application.

(3) This system has considered various impact factors to ensure the reliability of the route selection. Users can choose any factors, going by overlaying, primary Screening and optimization, to get the most suitable path.

\section{Design of index normalization}

Region stability calculation must be prior of the index normalization. This module includes the following functions: (1) building new index system; (2) preserving and updating index system; (3) normalizing every index, and building mapping relationship between layers and indexes. Based on these requirements, the specific implementation scheme is: (1) considering the existed landslides, mudslides, waterlogging in the database and expertise, screening the primary evaluation indexes; (2) removing a part of duplicate or cross indexes though correlation analysis, to keep the independence, only in this way, the influence factor of stability can be reflected entirely. (3) Determining the weight of each evaluation index by expert scoring and sensitivity analysis method. (4) Dividing every index into several items, and then confirming the value of every base on the influence in stability.

\section{Design of region stability evaluation}

Because of the huge discrepancy of internal and external stressed factors in different area, it is difficulty to calculate the stability than predict the time of disaster ${ }^{[6]}$. Think about the applicable condition, operability and data availability comprehensively, we choose the fuzzy comprehensive evaluation, multi-regress, and information method as the basic mathematical model to evaluate the stability, and then show the results in the form of unique-value thematic map.

The Specific implementation scheme is: (1) union of vector layers. We overlap all of the layers corresponding to influence factors, to obtain the result layer which includes the whole fields that every layer contains, and then extract the data which stability is known as samples, and the other is variable. (2) Choosing multiple mathematical solutions for stability evaluation analysis, and optimizing the parameters to make them more suitable.

According to the actual of Tianshan road, we divide the evaluation result into four levels, which is heavy risk, moderate risk, mild risk, non-risk, and use 4,3,2,1 to quantize and express. Here, we need to notice that the result is also an influence factor of route-selection.

\section{E. Design of route selection}

The factors that affect route strike are diverse ${ }^{[7]}$.
According to the technology roadmap, we can divide the selection into three stages: (1) original calculation. Based on the spatial analysis function, through multi-factors, multi-levels overlap and qualitative evaluation of raster cell, we can get several path schemes for choosing. (2) Quick screen. Comparing the generated route with actual condition, some alternatives were confirmed. (3) Recommending the best scheme. The final scheme was ascertained by evaluating the rest route in step 2. Based on these maids, the specific implementation scheme is:

(1) RSG reclassification. In the stage of route selection, we used some RSG data, such as stability result, slope and land use. In order to avoid the different dimensions and make every data comparable, the original data must be reclassified before overlap. The criterion of reclassification is the higher the level, the harder the passing-ability.

(2) RSG weighted overlay. In the stage of route selection, the contribution rate of every factor is different. According to the computational formula $\Sigma$ (weight $\times$ pixel value), the result of overlay stands for route cost of going by every cell.

(3) The best route generated. In the system design, the distance and direction cost are generated in background, and without temporary data. Using pre-generating cost data, the best route was got easily by inputting the beginning and end points.

\section{SYSTEM IMPLEMENTATION}

This research takes Tianshan road as the prototype. For decades, due to the spatial special geographic and climate conditions, Tianshan road is in the state of traffic block basically. For the sake of Xinjiang economy and carrying out of western development, it has been crucial to build new Tianshan road. As an important step, route-selection affects the quality and cost of project directly.

\section{A. Selection of evaluation index}

The whole system is divided into two main steps: the first is the stability evaluation analysis; the second is the route selection using the stability results. These two aspects all require necessary factor index. Aiming at the actual situation of Tianshan road, we have collected and vectored the topography and geological map along it, then built the spatial database: 1:50,000 contour, land use, bank, rainfall affect and distribution of disasters maps.

After cleaning up the existing data, we selected the land use, bank and distribution of disasters as evaluation index of stability, and selected slope, aspect and stability result as analysis index of route-selection. After confirming these indexes, all of them should be quantized.

\section{B. Stability evaluation and route-selection analysis}

In the stability evaluation, according to previous data, we selected 88 points as severe danger, 69 points as moderate danger, 26 points as mild danger and 53 points as non-risk. All of these points were regarded as the known samples. In accordance with the overlap result of index data, we can extract the unknown samples. In the route-selection analysis, according to expert experience method, the weight of land use, slope, ground fluctuation degree and stability 
results is set as $0.2,0.15,0.3$ and 0.35 , and the beginning and end points are $\mathrm{Du}$ shanzi and $\mathrm{Ku}$ che, the middle point is $\mathrm{Na}$ lati.

\section{Experiment and analysis}

Based on the above description, we have got the region stability along the Tianshan road using three kinds of mathematical models, and finally achieved the selected route successfully. Figure 2 was the result thematic map of stability by information model. Through the statistics data, we found that the scale of severe, moderate, mild and not-risk region is 4:5:2:3, which was generally consistent with the investigation result in the field. Figure 3 was the result of selected route, which reached the expectant target: priority to utilize the old line, and appropriate modification in partial section.

\section{CONCLUSIONS}

It has not been found before in the term of route selection based on region stability using ArcEngine. ArcEngine, adopting embedded development technology,

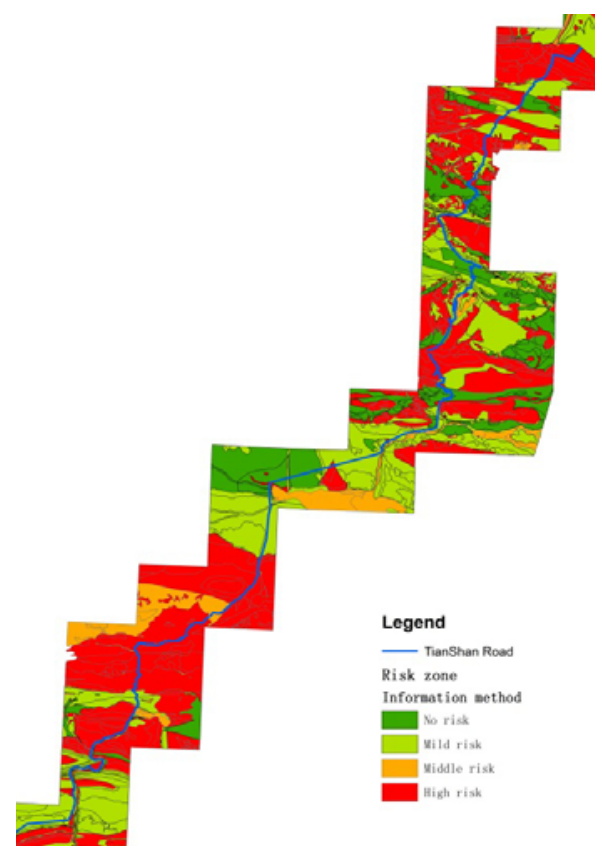

Figure 2. The theme of region stability analysis (Information Model)

\section{REFERENCES}

[1] Zhao Chun, Wang Wei, and Li Wei, "GIS-based decision support system for highway network management," Journal of transportation system engineering and information technology, vol.5, pp. 53-57, January 2005.

[2] Shu Endai, Li Ruimin, and Ma Zhaoyou, "Research and development of design-supporting system for expressway emergency rescue based on GIS," Bulletin of surveying and mapping, pp. 66-69, September, 2011.

[3] Ye Yali, Xu Jinliang, and Zhuang Chuanyi. "Highway route optimal based on Geological hazards risk evaluation," Highway, vol. 9, pp.11-16, September 2011 makes it easily that route selection combines with other information system. This design solves the problem of inefficiency, lower accuracy and weaker human-computer interaction, and is benefit to informationization, professionalization and scientization. The establishment of this system provides the necessary decision aid for policymakers, and is also a significant reference and lesson for the similar job. Of course, due to the optimization of model algorithm and the differences of selected index, there is some discrepancy in processing time, efficiency and accuracy, but author believes that these aspects will improve greatly later.

\section{ACKNOWLEDGMENT}

The authors are grateful to the foundation items: Key laboratory open-end fund of geo-special Information technology (KLGSIT2014-02); Key project of science and technology research in Henan department of education (14A420001).

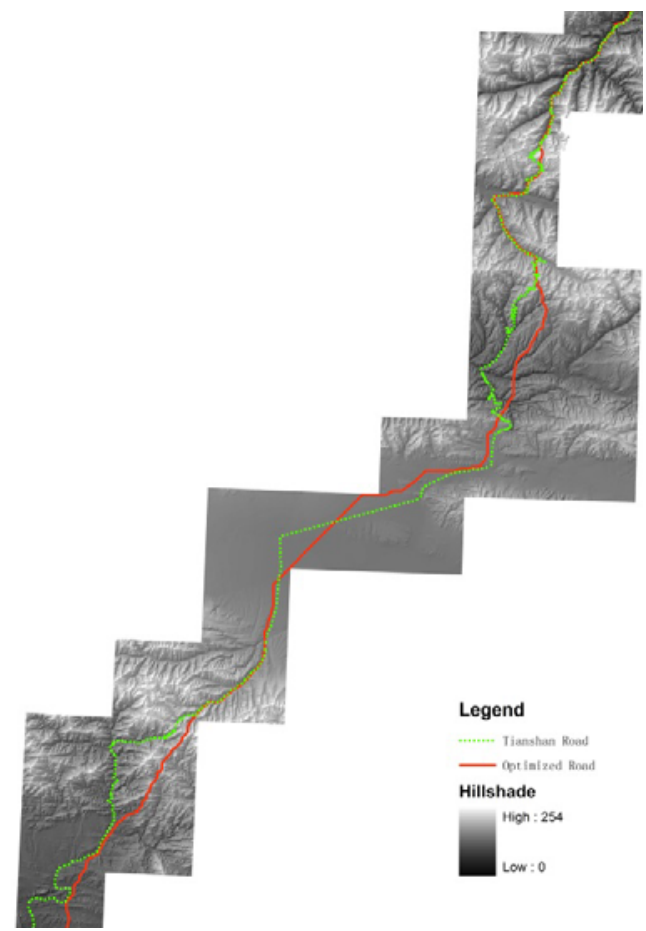

Figure 3. The final result graph of Route-selection

[4] Cheng Liang, Gong Jianya, and Liang Xinzheng, "Expressway maintenance decision support system based on GIS," Bulletin of surveying and mapping, pp. 69-72, June 2007.

[5] Zhao Zhonghua, Zhao Liming, and Zheng Jiangbo, "Highway management information system based on GIS," Journal of chang'an university(Natural science edition), vol. 25, pp. 69-72, January, 2005.

[6] Wang Yi, Guang Hong, and Jia Yonggang, "Application of GIS and SDSS to highway routing decision-making," Coastal engineering, vol. 20, pp. 31-37, January 2001.

[7] Ma Pingjun and Peng Xiaoping, "Design and implementation of highway geological hazards information management system based on GIS," Highway Engineering, vol. 34, pp. 37-40, March 2009. 